Supporting Information

\title{
Nanostructured Silicon Photocathodes for Solar Water Splitting Patterned by the Self-Assembly of Lamellar Block Copolymers
}

Lang Shen ${ }^{1}$, Chunlin $\mathrm{He}^{2}$, Jing Qiu ${ }^{1}$, Sung-Min Lee ${ }^{1}$, Abinasha Kalita ${ }^{1}$, Stephen B. Cronin $^{3}$, Mark P. Stoykovich ${ }^{2 *}$, Jongseung Yoon ${ }^{1,3^{*}}$

${ }^{1}$ Department of Chemical Engineering and Materials Science, University of Southern California, Los Angeles, California 90089, USA

${ }^{2}$ Department of Chemical and Biological Engineering, University of Colorado Boulder, Boulder, Colorado 80309, USA

${ }^{3}$ Department of Electrical Engineering, University of Southern California, Los Angeles, California 90089, USA

Correspondence and requests for materials should be addressed to J.Y. (js.yoon@usc.edu) or M.P.S. (mark.stoykovich@colorado.edu). 


\section{Supplementary Method}

\section{Semiempirical Numerical Modeling of BCP-patterned Silicon}

To elucidate optical effects of BCP lamellar nanopatterns implemented on silicon upon its absorption of standard solar radiation in water, semi-empirical numerical modeling based on finite-difference time-domain (FDTD) methods (Lumerical Solutions, Inc) was performed. In the software (FDTD Solutions 8.0), a model 3D nanostructured surface on semi-infinite silicon was created by importing top-view SEM images (Figure S2 and S3) of etched silicon with an area of $3.0 \times 5.0 \mu \mathrm{m}^{2}$. The imported 2D images were then extended vertically to form 3D structures (Figure S4), with heights same as those determined from cross-sectional SEM images at each etching time. FDTD calculations were performed for the semi-infinite silicon with a model nanostructured surface using periodic boundary conditions for $\mathrm{x}$ - and y-directions. A continuous plane-wave source with a broad Gaussian frequency spectrum (270 750 THz) was incident normally to the front surface of nanostructured silicon. For calculations of reflectance spectra with 1D photonic crystals at normal incidence as shown in Figure 3(c) and 3(d), 1D periodic gratings were implemented on the surface of semi-infinite silicon with periodicity and duty cycle identical to those obtained from experiments. The calculated spectra were averaged over transverse electric and transverse magnetic polarizations as schematically illustrated in Figure S6. 


\section{Supplementary Figures and Figure Captions}

(a)
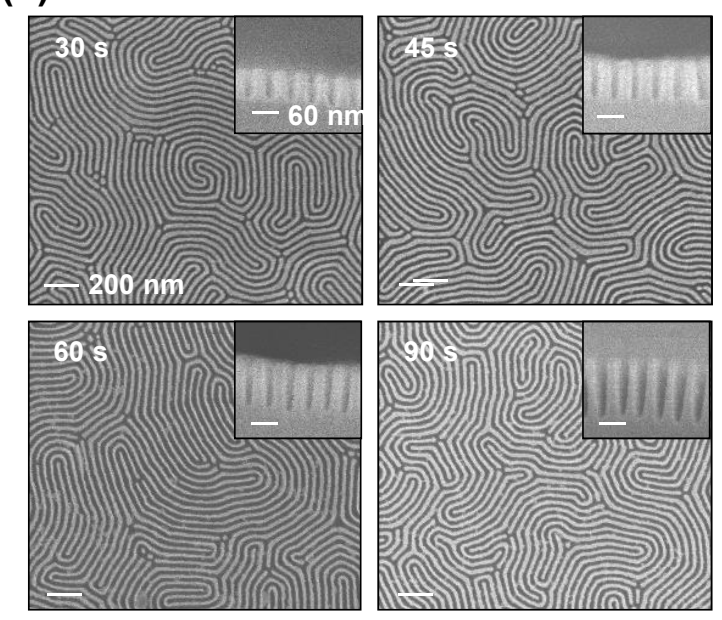

(c)

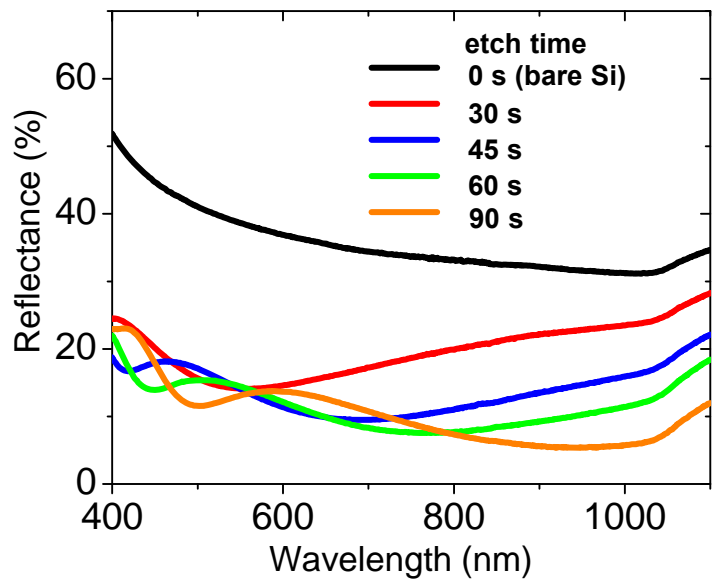

(b)

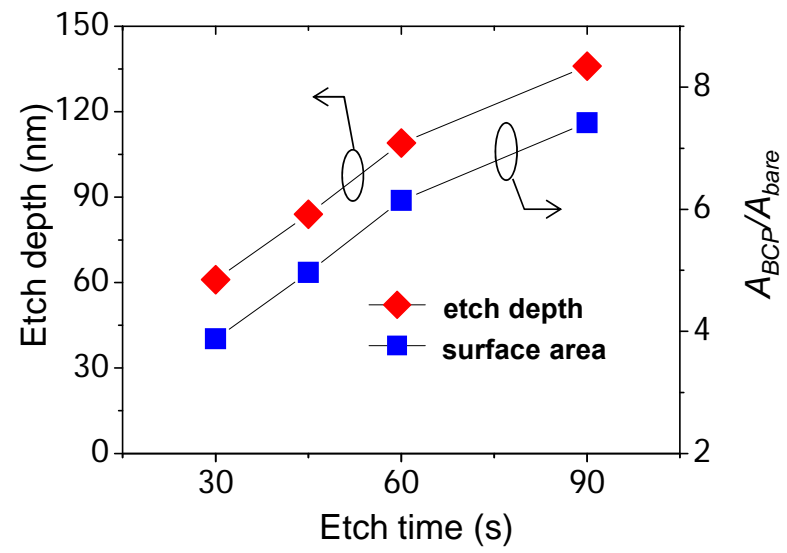

Figure S1: (a) Top-view SEM images of the nanostructured surface of the $n^{+} p^{+}-$silicon at various etching times The insets show cross-sectional-view SEM images. (b) Plot of the measured etch depth (red) and corresponding calculated ratio of the surface area (blue) for the nanostructured $\mathrm{n}^{+} \mathrm{pp}^{+}$-silicon $\left(A_{B C P}\right)$ at various etching times with respect to that of a bare silicon substrate $\left(A_{\text {bare }}\right)$. (c) Measured total (specular + diffuse) reflectance spectra of nanostructured and bare silicon at various etching times, measured on a spectrophotometer equipped with an integrating sphere at near-normal incidence $\left(\theta=8^{\circ}\right)$ using Spectralon ${ }^{\circledR}$ as a $100 \%$ reflectance standard. 
(a)

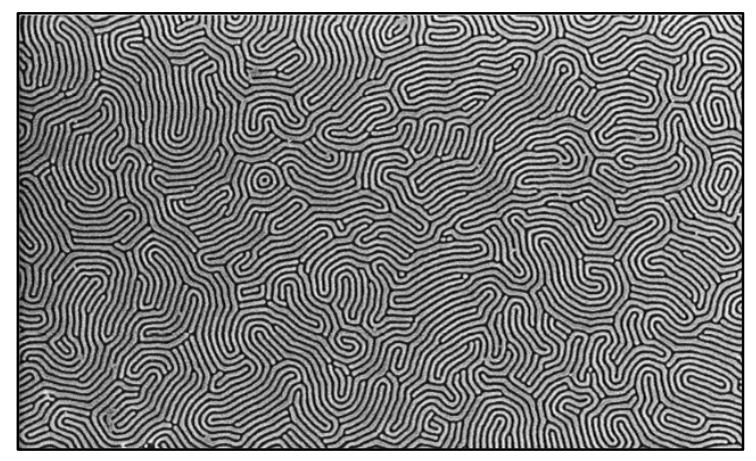

$1 \mu \mathrm{m}$

(c)

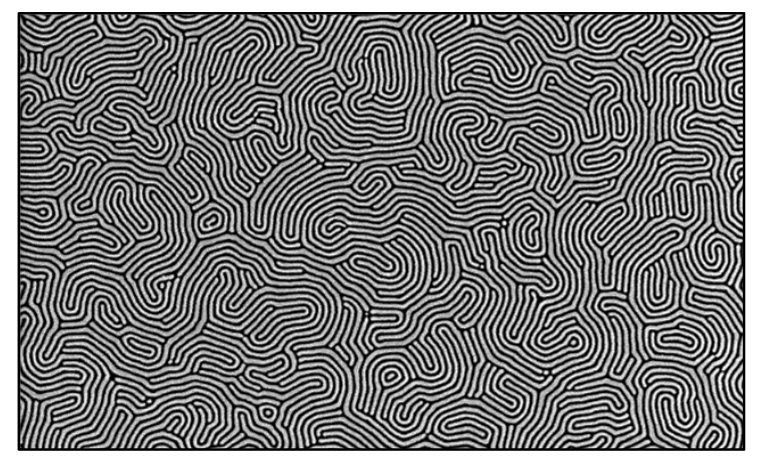

$1 \mu \mathrm{m}$ (b)
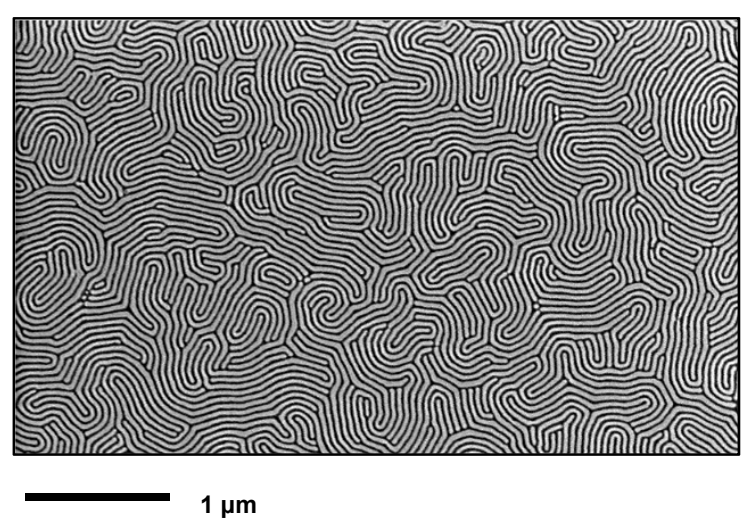

(d)

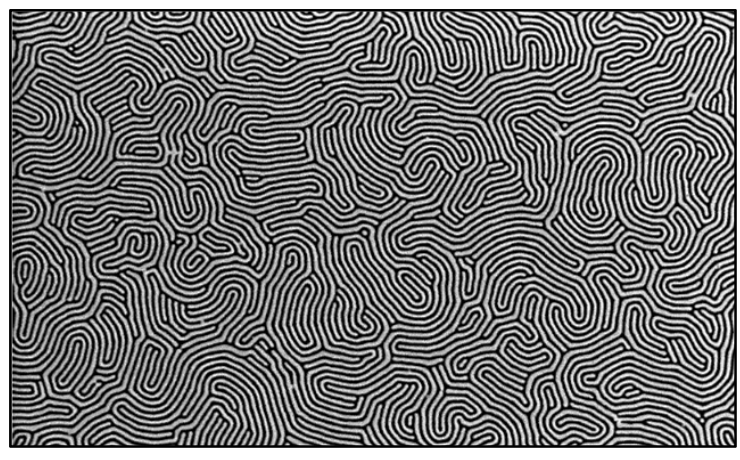

$1 \mu \mathrm{m}$

Figure S2: Top-view SEM images of $\mathrm{pp}^{+}$-silicon used for the construction of model nanostructured silicon for FDTD calculations, at the etching times of (a) 30, (b) 45, (c) 60, and (d) $90 \mathrm{~s}$, respectively. 
(a)

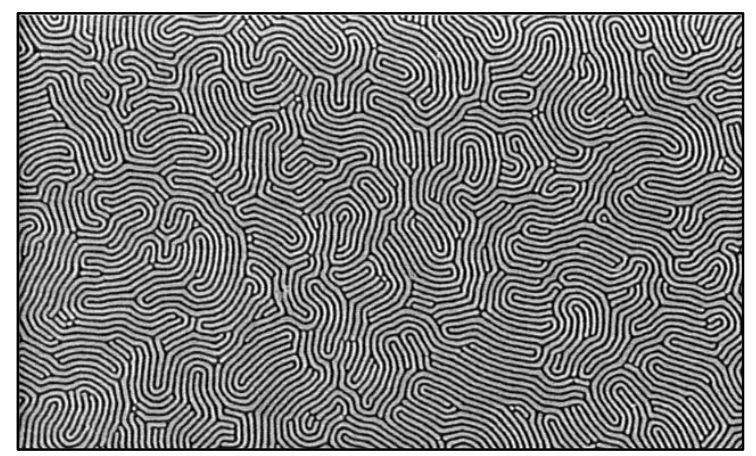

$1 \mu \mathrm{m}$

(c)

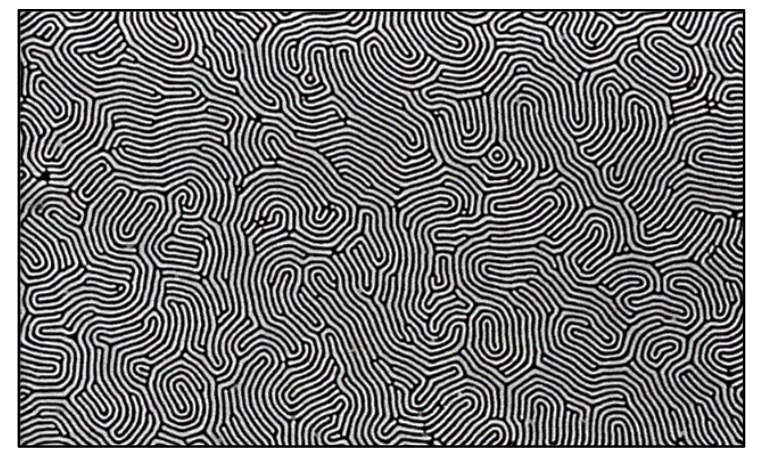

$1 \mu \mathrm{m}$ (b)

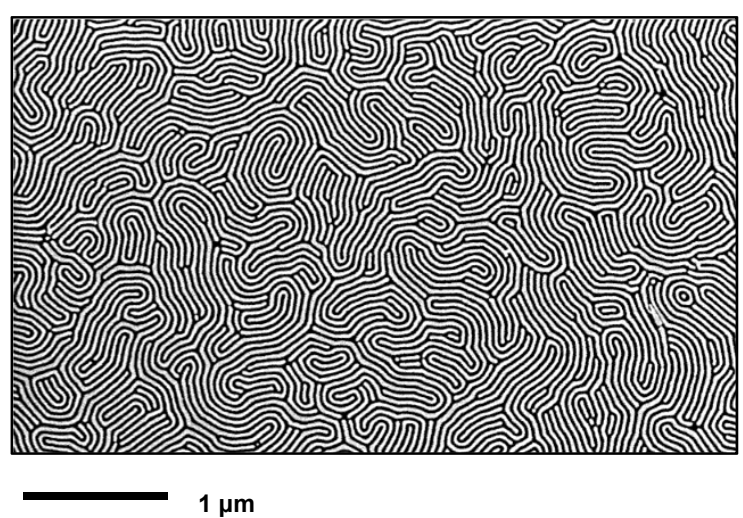

(d)

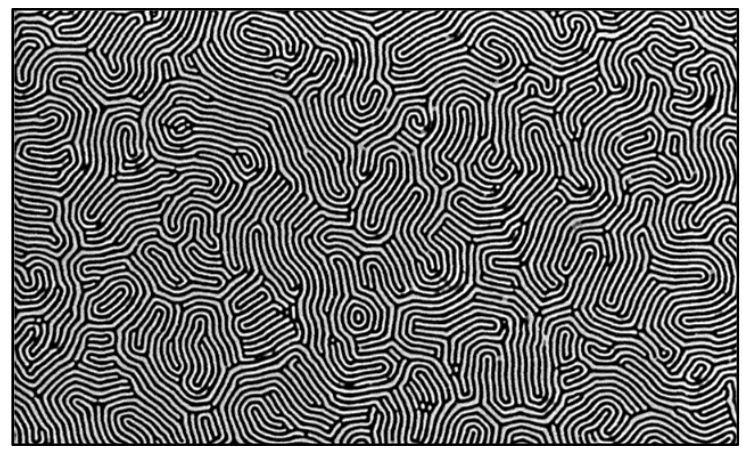

$1 \mu \mathrm{m}$

Figure S3: Top-view SEM images of $\mathrm{n}^{+} \mathrm{pp}^{+}$-silicon used for the construction of model nanostructured silicon for FDTD calculations, at the etching times of (a) 30, (b) 45, (c) 60, and (d) $90 \mathrm{~s}$, respectively. 
(a)

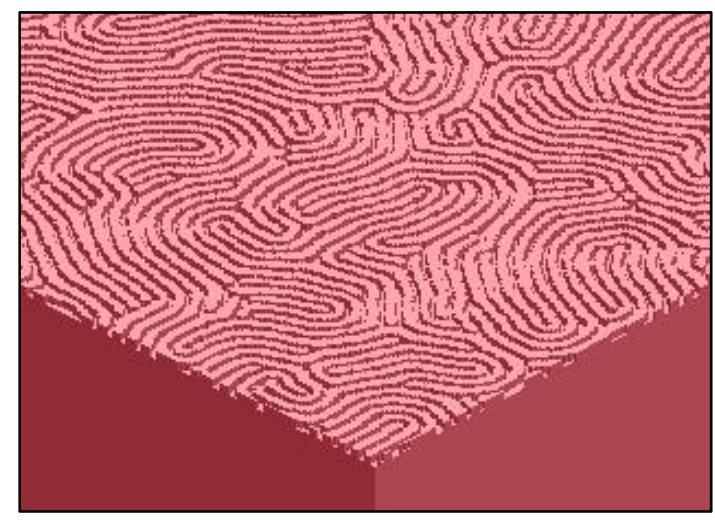

500 nm

(c)

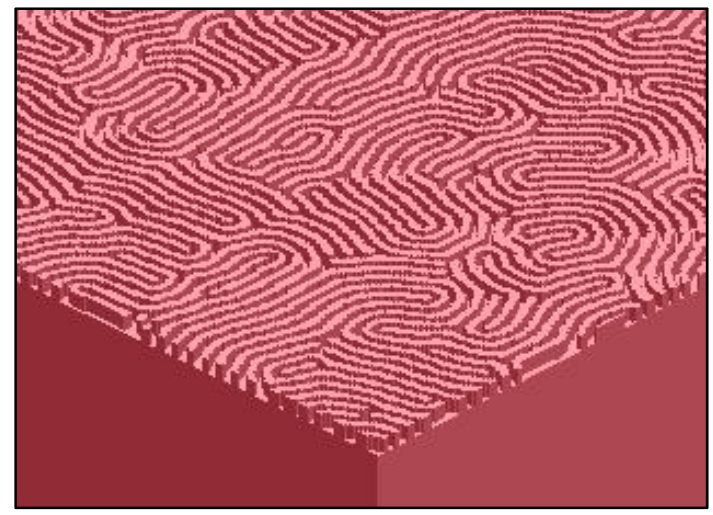

$500 \mathrm{~nm}$ (b)

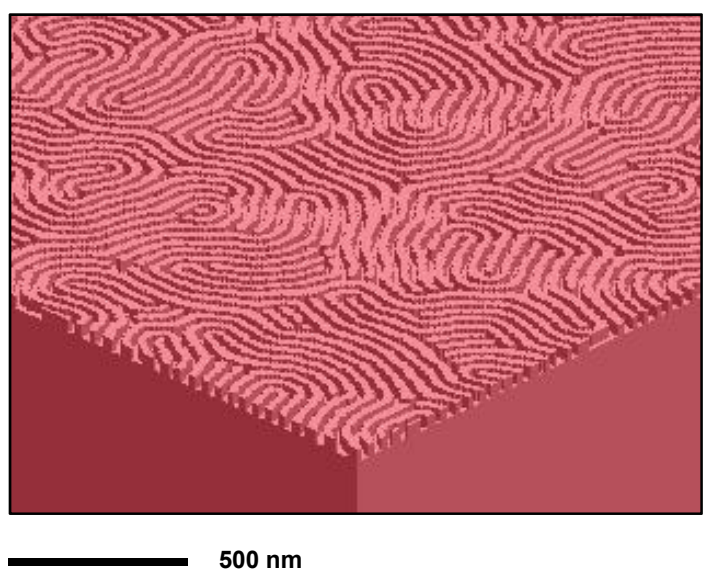

(d)

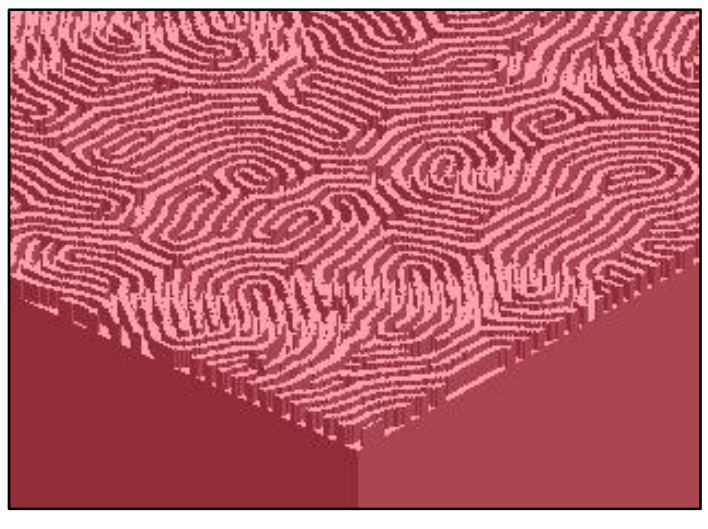

$500 \mathrm{~nm}$

Figure S4: Schematic illustration of constructed model 3D nanostructures for $\mathrm{pp}^{+}$-silicon with the characteristic surface morphologies of self-assembled BCP lamellae, at the etching times of (a) 30, (b) 45, (c) 60, and (d) $90 \mathrm{~s}$, respectively. 
(a)

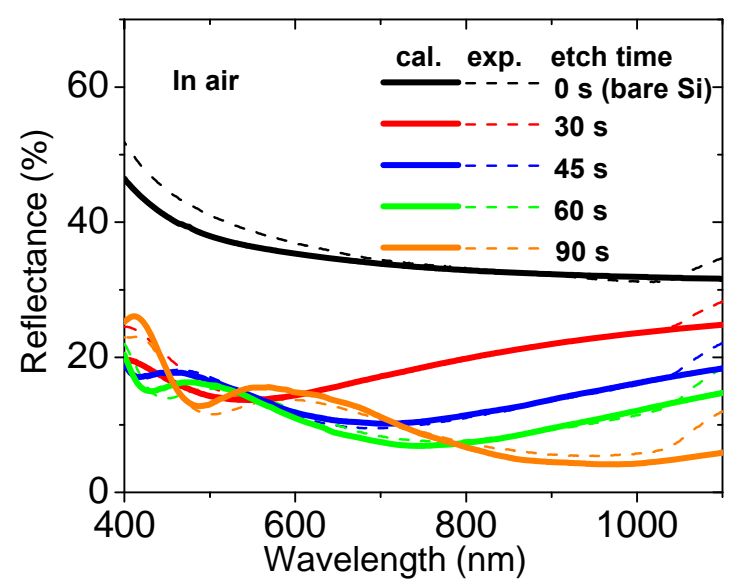

(c)

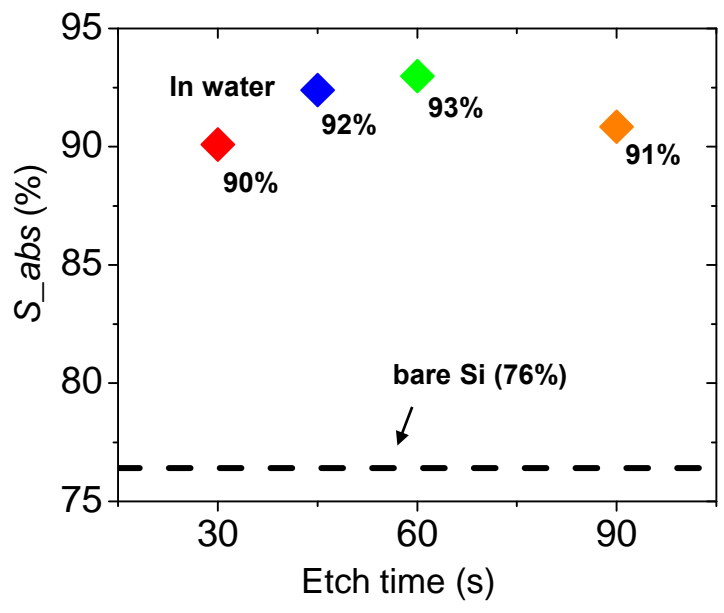

(b)

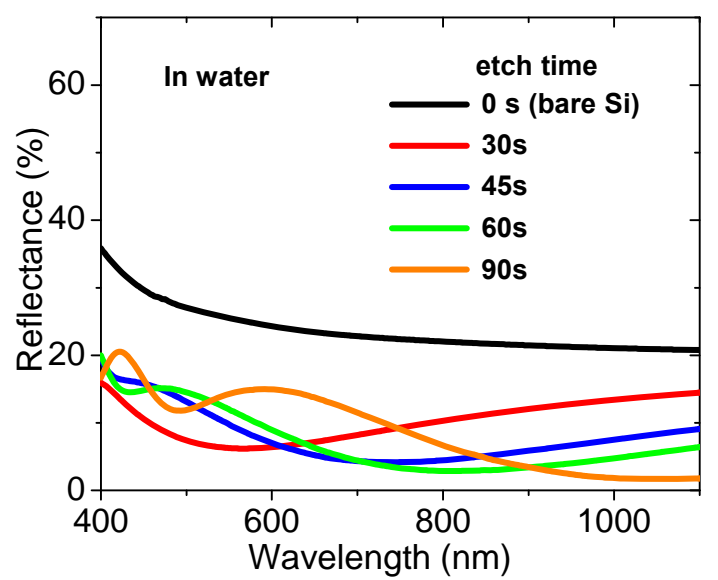

Figure S5: (a) Simulated reflectance spectra (solid lines) of $n^{+} p^{+}$silicon at normal incidence from semi-infinite silicon with model nanostructured surfaces of BCP lamellae at various etching times, overlaid with experimentally measured spectra (dotted lines) for comparison. (b) Simulated reflectance spectra from the same nanostructured silicon as in (a) yet in water as a superstrate medium. (c) The corresponding integrated solar flux absorption (S_abs) calculated by Eq. (1) over AM1.5G standard solar illumination. 


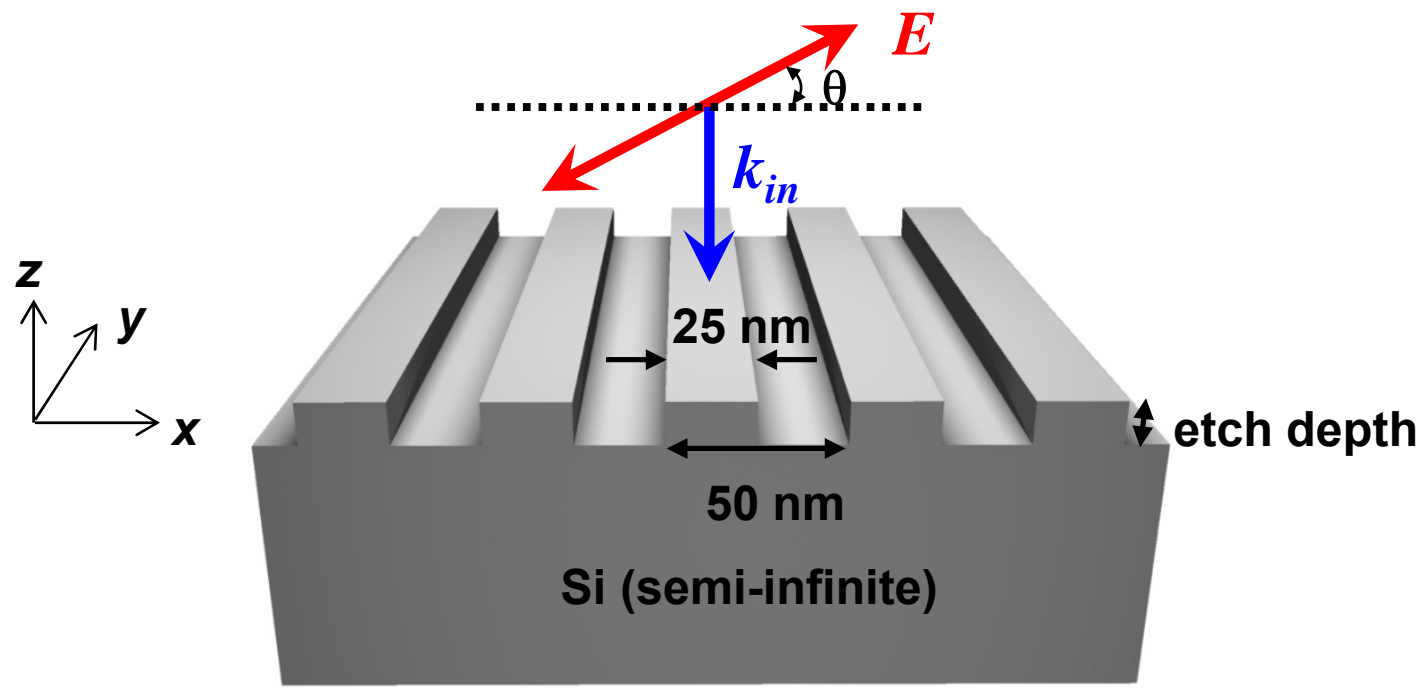

Figure S6: Schematic illustration of the model nanostructured silicon with 1D periodic gratings for the reflectance calculation by a FDTD method, where the periodicity and duty cycle of gratings are $50 \mathrm{~nm}$ and $50 \%$, respectively. The calculation was averaged over both transverse electric (i.e. electric field parallel to the $y$-axis, $\theta=90^{\circ}$ ) and transverse magnetic (i.e. electric field parallel to the $\mathrm{x}$-axis, $\theta=0^{\circ}$ ) polarizations. 